\title{
Erratum
}

\section{Cyclic Aromatic Analogues of the Hendrickson Reagent; NMR Studies and Electrophilic Properties}

Ziad Moussa* Synthesis 2012, 460.

The advance online publication (e-First) version of this article contained an error in Table 1, entry 1 . The yield under Method B was given as $5 \%$ but should be $85 \%$.

This mistake has been corrected for both the current online version and the print publication. 